\title{
TÓPICOS EN INVESTIGACIÓN CLÍNICA: LA PREGUNTA DE INVESTIGACIÓN
}

Ingrid Arévalo R.*, Merideidy Plazas V.**

\section{Resumen}

El proceso de la investigación en epidemiología requiere el reconocimiento de los elementos que lo constituyen, con el fin de alcanzar un desarrollo exitoso del mismo. Algunos de estos temas no son de fácil abordaje para el investigador clínico, que necesita guías claras para lograr reflejar sus preguntas en estudios clínicos. El siguiente artículo es el primero de una serie de documentos que presentarán los tópicos más importantes en investigación y epidemiología clínica iniciando con el tema de la pregunta de investigación.

Palabras clave: investigación biomédica, estudios epidemiológicos, proyectos de investigación, pregunta de investigación.

\section{TOPICS ON CLINICAL RESEARCH: THE RESEARCH QUESTION}

\section{Abstract}

Achievement of a succesful epidemiological research process requires knowledge on the elements comprised in it. Some of these matters are not easy to approach by the clinical researcher who needs clear guidelines to be able to reflect his questions in clinical trials. This article is the first of a series of documents that will present major topics in research and clinical epidemiology starting with the research question.

Key words: biomedical research, epidemiological studies, research projects, research question

La pregunta de investigación plantea en forma de interrogante el tema escogido. ${ }^{1}$ Por tanto, constituye la primera línea en la construcción y desarrollo de un estudio exitoso. Sumada al diseño, las variables y el procedimiento, la pregunta expone un problema que debe ser resuelto con el fin de obtener algún tipo de beneficio en la práctica clínica cotidiana. ${ }^{2}$ Resultarán otros que se presentan al consultar, analizar o referenciar literatura científica, cuando la pregunta de investigación puede verse reflejada en varias partes del artículo terminado: en la introducción, en forma de problema y con su justifica- ción; en el título y en el objetivo general; también es posible verla como resultado y conclusión que ofrecen respuestas al interrogante inicial. $^{3}$

Con frecuencia esta pregunta surge de situaciones donde se cuenta con amplio conocimiento sobre el tema o en la lectura crítica de nuevos artículos, avances y descubrimientos clínicos. ${ }^{4}$ Sin embargo, las más interesantes surgen del quehacer diario en la práctica, ante la presencia de uno o una serie de pacientes que nos dejan con interrogantes. ${ }^{5}$
Fecha recibido: Julio 28 de 2008 - Fecha aceptado: noviembre 10 de 2008

* MSc en epidemiología clínica. Instructora Asociada de Epidemiología. División de Investigaciones. Fundación Universitaria de Ciencias de la Salud, Hospital de San José, Bogotá D.C. Colombia.
** Especialista en Epidemiología. Profesora Asociada de Epidemiología. División de Investigaciones. Fundación Universitaria de Ciencias de la Salud, Bogotá D.C.Colombia. 
Los temas de los que trata son variados. Pueden ser acerca de la frecuencia de un evento (nuevo o presente), el riesgo de enfermar por una causa determinada (o de mejorar por la misma), la capacidad de diagnosticar una enfermedad (frente al verdadero diagnóstico), el pronóstico de los ya enfermos (mejoría o mortalidad), la utilidad de una intervención (condiciones controladas o naturales), los costos asociados con varias terapéuticas (su relación con el desenlace), la funcionalidad y calidad de vida (luego de una intervención) o la recopilación de evidencia sobre determinada entidad (para reglamentar la práctica diaria sobre evidencia o conocer la historia natural de la misma). ${ }^{6,7,8}$ Es de anotar que una buena pregunta no se construye en un solo momento; es a menudo una labor que se comparte con un equipo de trabajo y que evoluciona en la medida en que contemos con nueva información sobre el problema, con críticas constructivas acerca de su pertinencia, y con tiempo y experticia suficiente para expresar en palabras lo que en realidad nos cuestiona. ${ }^{9}$

A continuación examinaremos algunos factores que debemos tener en cuenta para lograr un comienzo acertado. Una buena pregunta se caracteriza por lo menos por tres elementos. En primer lugar, evitar el lenguaje ambiguo y las generalidades en su planteamiento ("mejoría", "niños", "sentirse bien"). En segundo lugar, versar sobre cuestiones específicas y limitadas, definiendo en forma adecuada el tema que está tratando ("sobrevida a cinco años", "fracturas tibiales tipo Schatzker I"). En tercer lugar, establecer límites espaciales y temporales, así como las unidades de observación que tomará ("unidades de cuidado intensivo con más de cuatro camas", "admisiones durante el mes de agosto del 2008").

La mayoría de las preguntas pueden dividirse en cuatro partes: el qué, comparado con qué, en quiénes y para qué. El qué identifica cuál es el tema que vamos a explorar (infecciones nosocomiales enero/2007, elevación del segmento ST, hemodiálisis), en otras palabras la exposición. El comparado con qué presenta, si es el caso, contra que se va a comparar el grupo anterior (infecciones nosocomiales enero/2008, síntomas clínicos, diálisis peritoneal) que nos lleva a pensar en tener dos grupos de estudio, aunque no todas las preguntas de investigación buscan necesariamente comparar. Quiénes nos muestra la población específica para la medición o donde inte- resa responder la pregunta (UCI, adultos mayores de 50 años, pacientes con insuficiencia renal crónica). Para qué, indica el desenlace que nos interesa mostrar (disminución de la incidencia, nuevos eventos de infarto agudo del miocardio, trasplante de riñón).

Además de estas cuatro partes básicas también es importante considerar cuándo y dónde. Cuándo indica el tiempo en el cual se recolectará la información que contestará la pregunta de investigación (enero del 2008, entre 2000 y el 2010). Dónde ubica al lector en la institución, municipio o departamento en el cual se desarrollará el trabajo de investigación (Hospital de San José, colegios públicos de la ciudad de Bogotá).

En esta forma, al realizar una pregunta de investigación se deben tener en cuenta las partes (qué, quiénes, para qué, comparado con qué, dónde y cuándo) y la intencionalidad del investigador. ${ }^{10}$ Cuando este pretende caracterizar una población, describir la frecuencia de una patología o documentar los síntomas y signos de un síndrome poco frecuente, se construyen preguntas descriptivas. En estas por lo regular se estudia un solo grupo de personas como en el siguiente caso: ¿Cuál es la frecuencia de trastorno depresivo mayor en los pacientes que asistirán a consulta externa de medicina interna en el Hospital de San José de Bogotá en el 2010?

En esta pregunta se identifican qué, trastorno depresivo mayor; comparado con qué, el interés del investigador no es comparar con otro grupo ni con otra patología; quiénes, pacientes que asisten a la consulta externa de medicina interna (un solo grupo); para qué, determinar la frecuencia; dónde, Hospital de San José; cuándo, en el 2010.

Por otra parte el investigador puede tener como intención buscar una asociación entre una exposición y un desenlace o mirar el riesgo de enfermedad en un grupo de pacientes comparado con otro. Con estas preguntas es importante destacar que se estudiará una o más exposiciones, uno o más desenlaces, una población en tiempo y espacio, y en dos o más grupos de pacientes. En este caso se construirán preguntas inferenciales, las cuales nos ayudan a tomar decisiones o a entender cómo y cuándo. ${ }^{10}$

Por ejemplo, en la siguiente pregunta: ¿Las pacientes con pre-eclampsia tuvieron mayor riesgo de presen- 
tar infección puerperal comparadas con las pacientes sin pre-eclampsia que asistieron a trabajo de parto en un hospital de tercer nivel de la ciudad de Bogotá en el 2005? ${ }^{11}$

Es una pregunta referente al riesgo de presentar infección post-parto en embarazadas sin infección debido a hipertensión inducida por el embarazo: qué, (exposición), presencia de pre-eclampsia; comparado con, ausencia de pre-eclampsia; en quiénes, embarazadas en trabajo de parto sin previa infección; para qué, (desenlace), frecuencia de infección puerperal; cuándo, 2005; dónde, un hospital de tercer nivel de la ciudad de Bogotá.

Cualquiera que sea el tema que trate nuestra pregunta, debemos evaluar los siguientes aspectos, con el fin de asegurar el éxito de nuestra investigación:

¿Nuestra pregunta es factible? ¿Tenemos la adecuada experticia para responderla nosotros mismos? ¿Hay recursos adecuados para abordar su respuesta? ¿Es suficiente la muestra de pacientes para responderla en forma adecuada?

¿Nuestra pregunta es interesante? ¿A quién interesaría la respuesta? ¿En qué aspectos influiría la posible respuesta? ¿Cambiaría nuestra forma de actuar en la práctica?

¿Nuestra pregunta es novedosa? ¿Produce la respuesta nueva información para la práctica? ¿Se dan otras perspectivas a un viejo problema? ¿Se muestran resultados más aplicables a nuestros pacientes?

¿Nuestra pregunta sigue principios éticos? ¿El procedimiento para responder la pregunta produce riesgos inaceptables? ¿Se suprimen derechos básicos? ¿Se tiene aprobación de un comité de ética institucional?

¿Nuestra pregunta es relevante? ¿Genera nuevos aportes al conocimiento sobre la entidad estudiada? ¿Tiene impacto clínico? ¿Es importante para la sociedad? ¿Es guía para futuras investigaciones?

Habrá un buen inicio si podemos responder de manera afirmativa la mayor parte de los anteriores cuestionamientos. Se recomienda examinar todos estos elementos a medida que se tengan nuevos avances en el planteamiento, ya que no solo inciden en la delimitación del problema de investigación, sino en otros como el diseño a emplear, el tiempo del estudio, el presupuesto, entre otros, que serán analizados en posteriores artículos.

\section{Referencias}

1. Cummings SR, Browner WS, Hulley SB. Conceiving the research question. En: Hulley SB, Cummings SR, Grady D, Browner WS, Hearst N, Newman T, et al, editors. Designing clinical research. 2nd ed. Baltimore: Williams \& Wilkings; 2001.

2. Sackett DL. Clinical epidemiology: what, who and whiter. J Clin Epidemiol. 2002 Dec; 55(12):1161-6.

3. Londoño JL. Metodología de la investigación epidemiológica. 3a. ed. Bogotá: Manual Moderno; 2006.

4. Guyatt GH, Rennie D. Users' guides to the medical literature: a manual for evidence-based clinical practice. Chicago: American Medical Association; 2001.

5. Sackett D, Straus SE, Richardson WS, Rosenberg W, Haynes R. Asking answerable clinical questions. En: Sackett D, Straus SE, Richardson WS, Rosenberg W, Haynes R, editors. Evidence-based medicine: how to practice and teach EBM. 2nd ed. China: Churchill Livingstone; 2000. p. 13-27.

6. Fletcher RH, Fletcher SW. Clinical epidemiology: the essentials. 4th ed. Baltimore: Lippincott Williams \& Wilkins; 2005.

7. Sackett D, Haynes R, Tugwell P, Guyatt G.H. Clinical epidemiology: a basic science for clinical medicine. 2nd ed. Baltimore: Lippincott Williams \& Wilkins; 1991.

8. Lozano León JM. Investigación en salud: qué es y para qué sirve. En: Morales Ruiz Á, Morillo Zárate LE, editores. Epidemiología clínica. Bogotá: Medica Panamericana; 2004. p. 19-28.

9. Morillo Zárate LE. Preparación de una propuesta de investigación y de un protocolo. En: Morales Ruiz Á, Morillo Zárate LE, editores. Epidemiología clínica. Bogotá, Buenos Aires: Médica Panamericana; 2004. p. 67-84.

10. Ruiz JG. La pregunta de investigación. En: Morales Ruiz Á, Morillo Zárate LE, editores. Epidemiología clínica. Bogotá, Buenos Aires: Médica Panamericana; 2004. p. 29-50.

11. Gaitán H, Gomez PI. Association between pregnacyinduced hypertension and Post-partum infection in the Instituto Materno Infantil. Bogota, Colombia. Casecontrol study. Rev. Colomb. Obstet. Ginecol. 2004; 55(3):193-200. 